\title{
Alpha-Beta Weightless Neural Networks
}

\author{
Amadeo José Arguelles-Cruz, Itzamá López-Yáñez, \\ Mario Aldape-Pérez, and Napoleón Conde-Gaxiola \\ IPN Centro de Investigación en Computación \\ Juan de Dios Bátiz s/n esq. Miguel Othón de Mendizábal \\ Unidad Profesional Adolfo López Mateos \\ Del. Gustavo A. Madero, México, D.F. México \\ jamadeo@cic.ipn.mx, ilopezyb05@ipn.mx, maldape@gmail.com
}

\begin{abstract}
A novel weightless neural network model is presented, based on the known operations Alpha and Beta, and three original operations proposed. The new model of weightless neural network has been called CAINN - Computing Artificial Intelligent Neural Network. The experimental aspect is presented by applying the CAINN model to several known databases. Also, comparative studies about the performance of the CAINN model concerning ADAM weightless neural network and other models are reported. Results exhibit the superiority of the CAINN model over the ADAM model, its counterpart as a weightless neural network; and over other models immersed in the state of the art of neural networks; taking into account the No Free Lunch theorem.
\end{abstract}

Keywords: Weightless Neural Networks, ADAM, CAINN, Classifier, No Free Lunch.

\section{Introduction}

Weightless neural networks (WNNs) are computational models, derived from the artificial neural networks development, and used in pattern recognition tasks. They share the characteristic of receiving at their inputs and providing at their outputs binary values. The functions carried out by each neuron are contained into look-up tables made up by random access memory (RAM).

Bledsoe and Browning reported in 1959 a method called $n$-tuple [1. This method is based on distributed computation, it uses one-shot memorization task, and has among its properties simplicity of learning by memorization and speed of operation. Aleksander used $n$-tuple sampling machines to be applied on adaptive neural networks [2]-4. Then, higher capacity and low cost RAM based memories appeared, stimulating the development of higher capacity machines [5]. Later on, very important researches developed WNNs models that were extensions to the $n$-tuple theory and use other techniques in the classification of objetcs [6]-[12].

In this paper, we focus on the creation, design, and test of a new weigthless neural network called Alpha-Beta Weightless Neural Networks, and helped by using the Alpha-Beta associative memories [13. The Method section covers formally the new operations, as well as the learning and recovering phases of 
the Alpha-Beta weightless neural network algorithm. The Results section shows the performance and comparison of ADAM and CAINN algorithms over two databases contained in the UCI Machine Learning Repository [16] for recovery and classification, and the comparison in pattern classification between CAINN and other classifiers. The last section of this work is dedicated to discussing the relevance and behavior of CAINN when compared to other classifiers, taking into account the No Free Lunch theorem effects [18]-[19].

\section{Method}

This section contains original definitions and operators used in the Alpha-Beta weightless neural networks model, as well as the CAINN algorithm.

\section{$2.1 \alpha_{g}$ Operation}

In order to characterize the recalling phase of the new neural network model, by using Alpha and Beta operations [13], a new operation is required, which is called Generalized Alpha, denoted with the symbol $\alpha_{g}$. As its name suggest, this operation is a generalization of the original Alpha binary operation, so that it accepts real values as arguments, handing over to the output values in the set $B=\{00,01,10\} ;$ namely $a_{g}=\mathbb{R} \times \mathbb{R} \longrightarrow B$, an defined as follows:

Let $x \in \mathbb{R}, y \in \mathbb{R}$ be any two real numbers, then

$$
\alpha_{g}= \begin{cases}00 & \text { if } x<y \\ 01 & \text { if } x=y \\ 10 & \text { if } x>y\end{cases}
$$

\section{$2.2 \sigma_{\alpha}$ and $\sigma_{\beta}$ Operations}

Both operations, which will be used in learning and recalling phases of the model described in the following section, are defined as follows:

Sigma-Alpha operation: $P_{m \times r} \quad \sigma_{\alpha} \quad Q_{r \times n}=\left|f_{i j}^{\alpha}\right|_{m \times n}$, where $f_{i j}^{\alpha}=\sum_{k=1}^{r} \alpha\left(p_{i k}, q_{k j}\right)$

Sigma-Beta operation: $P_{m \times r} \quad \sigma_{\beta} \quad Q_{r \times n}=\left|f_{i j}^{\beta}\right|_{m \times n}$, where $f_{i j}^{\beta}=\sum_{k=1}^{r} \beta\left(p_{i k}, q_{k j}\right)$

Given column vectors $x \in A^{n}$ and $y \in A^{m}$, with $A=\{0,1\}$, and the $m \times n$ dimension matrix $y \sigma_{\alpha} x^{t}$, the $i j$-component of such matrix is:

$$
\left[y \sigma_{\alpha} x^{t}\right]_{i j}=\alpha\left(y_{i}, x_{j}\right)
$$

The following is the case of an array with dimensions $m \times n$ and a column vector with $n$ dimension uses $\sigma_{\beta}$ operation It provides a colum vector of $m$ dimension, which $i$-component is described as follows:

$$
\left(\mathbf{P}_{m \times n} \sigma_{\beta} \mathbf{x}\right)_{i}=\sum_{j=1}^{n} \beta\left(p_{i j}, x_{j}\right)
$$

Similar results are obtained for the operation $P_{m \times n} \sigma_{\alpha} x$. 


\subsection{Alpha-Beta Weightless Neural Network Algorithm}

This new model, denoted as CAINN (Computer Artificial Intelligent Neural Network), takes the set $A=\{0,1\}$ where input and output patterns are coming from, and consider the fundamental set $\left\{\left(\mathbf{x}^{\mu}, \mathbf{y}^{\mu}\right) \mid \mathbf{x}^{\mu} \in A^{n}, \mathbf{y}^{\mu} \in A^{m}\right\}$ where $\mu=1,2 \ldots, p$.

A class code is applied in every input and output pattern presented at the learning and recalling phases, named $z^{\mu} \in A^{k}$, where $k$ is a one-hot dimension vector representing the binary pattern decimal value $x^{\mu}$.The algorithm is described as follows:

\section{CAINN Learning Phase}

1. Two null matrices are created: $\mathbf{P}=\left[p_{i j}\right]_{k \times n}$ and $\mathbf{Q}=\left[q_{i j}\right]_{m \times k}$.

2. Every component presented in $\mathbf{P}$ and $\mathbf{Q}$ is denoted as $p_{i j}(0)$ and $q_{i j}(0)$ respectively.

3. Every component $p_{i j}$ and $q_{i j}$ is modified using the following rules:

(a) Class code $z^{\mu}$ with $k$ dimension is proposed.

(b) Matrix $\mathbf{P}$ is updated acording to the following rule:

$$
p_{i j}(\mu)=\beta\left(\alpha\left[p_{i j}(\mu-1), 0\right], \beta\left(z_{i}^{\mu}, x_{j}^{\mu}\right)\right)
$$

(c) In a similar manner, $\mathbf{Q}$ is updated acording to the following rule:

$$
q_{i j}(\mu)=\beta\left(\alpha\left[p_{i j}(\mu-1), 0\right], \beta\left(y_{j}^{\mu}, z_{i}^{\mu}\right)\right)
$$

4. An additional column vector, $s \in A^{n}$, is generated. Such column vector has at their $i$-component the sum of all the positive values presented in the $i$-row of matrix $\mathbf{P}$, then:

$$
s_{i}=\sum_{j=1}^{k} p_{i j}, \text { such that } p_{i j}>0
$$

CAINN Recalling Phase. An input pattern $\tilde{\mathbf{x}}^{\omega} \in A^{n}$, where $\omega \in\{1,2, \ldots, p\}$, is considered. The following actions are applied over the input pattern:

1. The operation $\mathbf{P} \sigma_{\beta} \tilde{\mathbf{x}}^{\omega}$ is applied, this means:

$$
\left(\mathbf{P} \sigma_{\beta} \tilde{\mathbf{x}}^{\omega}\right)_{i}=\sum_{j=1}^{n} \beta\left(p_{i j}, \tilde{x}_{j}^{\omega}\right), \forall i \in\{1,2, \ldots, k\}
$$

2. A transition vector $t^{\omega}$ for class code $z^{\omega}$ is computed as follows:

$$
t_{i}^{\omega}=\alpha_{g}\left(\sum_{j=1}^{n} \beta\left(p_{i j} \tilde{x}_{j}^{\omega}\right), \vee\left[\sum_{j=1}^{n} \beta\left(p_{h j} \tilde{x}_{j}^{\omega}\right)\right]\right), \forall i \in\{1,2, \ldots, k\}
$$


3. The $H$ set is defined:

$$
H=\left\{h \mid \sum_{j=1}^{n} p_{h j} \tilde{x}_{j}^{\omega}=\bigvee_{i=1}^{k}\left(\sum_{j=1}^{n} p_{i j} \tilde{x}_{j}^{\omega}\right)\right\}
$$

4. Class code $z^{\omega}$ is computed:

$$
z_{i}^{\omega}=\beta\left\{\alpha_{g}\left(t_{i}^{\omega}, 1\right), \alpha_{g}\left[\bigwedge_{h \in H}\left(\sum_{j=1}^{n} p_{h j}\right), \sum_{j=1}^{n} p_{i j}\right]\right\}, \forall i \in\{1,2, \ldots, k\}
$$

5. Thereafter, the following operation must be computed:

$$
\left(\mathbf{Q} \sigma_{\beta} \mathbf{z}^{\omega}\right)_{i}=\sum_{j=1}^{k} \beta\left(q_{i j}, z_{j}\right), \forall i \in\{1,2, \ldots, m\}
$$

6. And finally, output vector is obtained:

$$
y_{i}^{\omega}=\alpha_{g}\left(\sum_{j=1}^{k} \beta\left(q_{i j}, z_{j}\right), \bigvee_{h=1}^{m}\left[\sum_{j=1}^{k} \beta\left(q_{h j}, z_{j}\right)\right]\right), \forall i \in\{1,2, \ldots, m\}
$$

\section{Results}

This section shows the application of the proposed algorithm to Iris Plants and Contraceptive Method Choice databases, both from UCI Machine Learning Repository [16]. The results are compared against those of ADAM, given that the proposed model is inspired on ADAM [9].

\subsection{Pattern Recall}

Complete Fundamental Set Recalling. The first estimate of the Alpha-Beta and ADAM weightless neural networks performance was made by learning and recalling of the fundamental set in its entirety. The process which was applied to both models is as follows: learning the first pattern and trying to recall it, learn the second pattern and try to recall the two learned, and so on until it has learned the entire fundamental set. Particularly, for the first dataset, 150 recall attempts per experiment were conducted while for the second dataset 1,473 recall attempts per experiment were made.

For a more reliable estimate of the recalling rate reached with both datasets, the procedure was carried out 50 times for each set, selecting randomly the order of patterns presented in the fundamental set. Thus, there were 7500 experiments done for the Iris Plant dataset, and 73,650 for the CMC dataset. The results presented in table 1 are obtained from the average of attempts to recover, for each set. 
Table 1. Recall rate average for complete datasets

\begin{tabular}{lcc}
\hline \multicolumn{3}{l}{ Method Iris Plant CMC } \\
\hline ADAM & $9 \%$ & $8 \%$ \\
CAINN & $100 \%$ & $100 \%$ \\
\hline
\end{tabular}

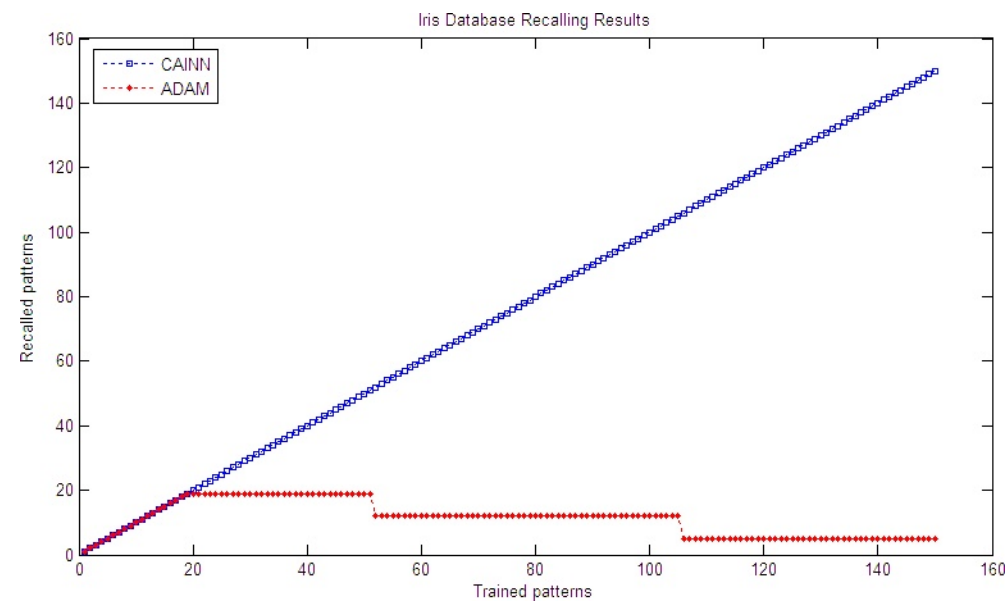

Fig. 1. Recalling index for first dataset

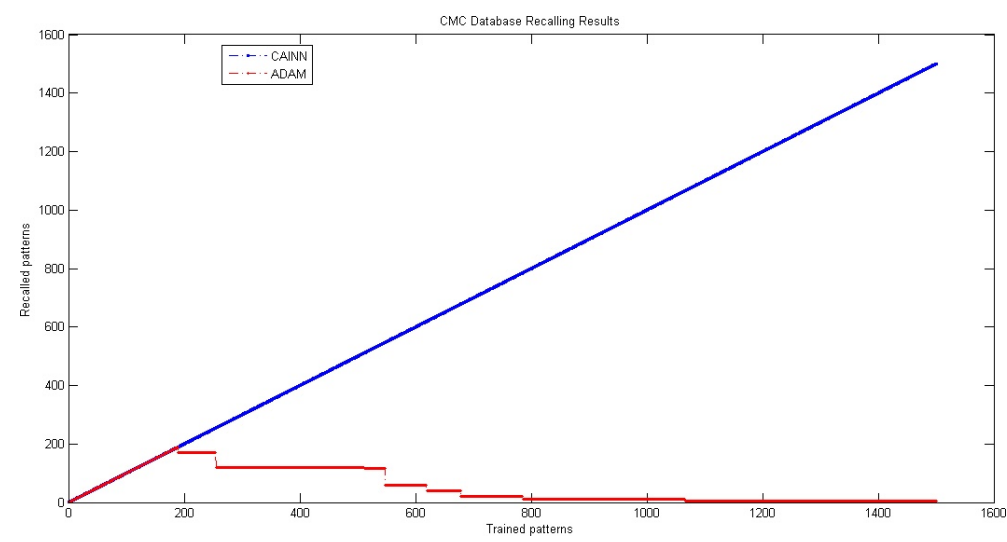

Fig. 2. Recalling index for second dataset

Clearly, the performance of the model proposed in the present work, is superior to that reached by the ADAM model, as is shown both in figures 1 and 2 .

Recall is considered correct when all the bits that comprise a pattern are being recalled; otherwise, it is considered incorrect. It is worth mentioning that, for both sets of data, the Alpha-Beta weightless neural network model achieves 
Table 2. Recall rate average for dataset partitionings of $70 / 30$

\begin{tabular}{ll}
\hline \multicolumn{2}{l}{ Method Iris Plant CMC } \\
\hline ADAM $17 \%$ & $12 \%$ \\
CAINN $74 \%$ & $72 \%$ \\
\hline
\end{tabular}

Table 3. Classification rate average for complete datasets

\begin{tabular}{lc}
\hline \multicolumn{2}{l}{ Method Iris Plant CMC } \\
\hline ADAM $70 \%$ & $65 \%$ \\
CAINN $100 \%$ & $100 \%$ \\
\hline
\end{tabular}

better performance in terms of recall rate. Also, it can be observed that the ADAM model suffers from problems of saturation. This causes ambiguity conditions over involved patterns on the recall phase, making their correct recall impossible.

Partial Fundamental Set Recalling. The second estimation of performance made to the Alpha-Beta weightless neural network model was carried out by learning $70 \%$ and recalling $30 \%$ of the fundamental set. This procedure was done 50 times for each test set, selecting randomly the patterns involved in each partition. Table 2 provides the results based on the recovery attempts average for each set, while figures.

\subsection{Pattern Classification}

Complete Fundamental Set Classification. For this phase, the same databases presented in the previous section were used. Each experiment was carried out in the following manner: first, there were taken in a random fashion the same number of input patterns for each class, obtaining a balanced test set. Afterwards, the whole dataset was taken for its classification. This procedure was carried out 50 times for each dataset. Table 3 provides the results based on the classification rate average for each test set.

Partial Fundamental Set Classification. The fourth and last estimation for the Alpha-Beta weightless neural network performance was done by learning the $70 \%$ of each fundamental set and classifying the remaining $30 \%$. This procedure was carried out 50 times for each dataset, selecting randomly the patterns involved in each partition. Results provided by table 4 are obtained from the the average of classification rate, over each dataset.

Table 4. Classification rate average for dataset partitionings of $70 / 30$

\begin{tabular}{lr}
\hline \multicolumn{2}{l}{ Method Iris Plant CMC } \\
\hline ADAM $58 \%$ & $42 \%$ \\
CAINN $89 \%$ & $81 \%$ \\
\hline
\end{tabular}


Table 5. Classification rate comparison

\begin{tabular}{lrc}
\hline \multicolumn{1}{c}{ Classifier } & \multicolumn{2}{c}{ Iris Plant CMC } \\
\hline ADAM & $58 \%$ & $42 \%$ \\
CAINN & $89 \%$ & $81 \%$ \\
RAMP [14] & $97 \%$ & - \\
STEP [14] & $77 \%$ & - \\
SVM [14], 15] & $96 \%$ & $54 \%$ \\
kNN [14], 15] & $96 \%$ & $48 \%$ \\
MLP [14, [15] & $95 \%$ & $53 \%$ \\
RM [15], & $97 \%$ & $55 \%$ \\
C4.5 [17] & $94 \%$ & $63 \%$ \\
C4.5+m [17] & $93 \%$ & $66 \%$ \\
\hline
\end{tabular}

The above results can be compared with those reported in the scientific literature. Table 5 includes results mentioned above, as well as those reported in 14] for Iris, and in [15] and [108] for Iris and CMC.

\section{Discussion}

From table 5 it is derived that CAINN classification rate overpasses the ADAM and STEP models in performance, for the Iris Plant dataset, presenting at the same time, advantages of up to $15 \%$ over the other classifiers on the CMC dataset.

Regarding the Iris Plant database, it is clear that CAINN far exceeds ADAM in the classification rate, surpassing also the neural network STEP. However, other classifiers present better performance (RAMP, SVM, kNN, MLP, RM, $\mathrm{C} 4.5$ and $\mathrm{C} 4.5+\mathrm{m}$ ), although CAINN comes close to their results. This drawback shown with regard to the last set of classifiers is overcome on the CMC database, where CAINN surpasses all classifiers included in the table by an extent margin (15\% difference with $\mathrm{C} 4.5+\mathrm{m}$, the classifier whose results most closely approximate those of CAINN).

This behavior is nothing strange, because the no Free Lunch Theorem [18]19. indicates that when an algorithm (of classification) is very good with some family of problems, must be not so good to others.

Is is also worth mentioning that all the algorithms presented at the table 5] which show a better performance than CAINN on the Iris Plant database, also have a greater complexity, several of them being even iterative. Thus, in spite of offering a lower classification rate than those classifiers (although by a margin of around 5\%), CAINN offers the advantages of being one-shot and relatively simpler.

Acknowledgements. The authors would like to thank the Instituto Polit écnico Nacional (Secretaría Académica, COFAA, SIP, and CIC), the CONACyT, and SNI for their economical support to develop this work. 


\section{References}

1. Bledsoe, W.W., Browning, I.: Pattern Recognition and Reading by machine. In: Proc. Eastern Joint Computer Conference, pp. 225-232 (1959)

2. Aleksander, I.: Canonical neural nets based on logic nodes. In: First IEE International Conference on Artificial Neural Networks, pp. 110-114. IEEE, London (1989)

3. Kan, W.K., Aleksander, I.: A probabilistic logic neuron network for associative learning. In: Proceedings of the IEEE First International Conference on Neural Networks, ICNN 1987, pp. 541-548 (1987)

4. Myers, C.E., Aleksander, I.: Output functions for probabilistic logic nodes. In: First IEE International Conference on Artificial Neural Networks, pp. 310-314 (1989)

5. Aleksander, I., Thomas, W.V., Bowden, P.A.: WISARD, a Radical Step Forward in Image Recognition. Sensor Review 4(3), 120-124 (1984)

6. Ludermir, T.B., et al.: Weightless Neural Models: A Review of Current and Past Works. Neural Computing Surveys 2, 41-61 (1999)

7. Austin, J.: RAM based neural networks, a short history. In: RAM-Based Neural Networks. World Scientific Publishing Co. Pte. Ltd, Singapore (1998)

8. Austin, J.: Grey scale n-tuple processing. Springer, Berlin (1988)

9. Austin, J.: ADAM: A Distributed Associative Memory for Scene Analysis. In: Caudhill, M., Butler, C. (eds.) Proceedings of First International Conference on Neural Networks, San Diego, CA, pp. 285-295 (1987)

10. Al-Alawi, R.: FPGA Implementation of a Pyramidal Weightless Neural Networks Learning System. International Journal of Neural Systems 13(4), 225-237 (2003)

11. Argüelles, A.J., Díaz De Leon, J.L., Yañez, C., Camacho, C.: Pattern recognition and classification using weightless neural networks and Steinbuch Lernmatrix. In: SPIE Optics and Photonics 2005. SPIE, pp. (59160)P1-P8 (2005)

12. Howells, G., Fairhurst, M.C., Rahman, F.: An exploration of a new paradigm for weightless RAM-based neural networks. Connection Science 12(1), 65-90 (2000)

13. Yáñez-Márquez, C.: Memorias Asociativas basadas en Relaciones de Orden y Operadores Binarios (in Spanish). Ph.D. Thesis. National Polytechnics Institute, Computers Research Center, Mexico (2002)

14. Tran, Q., Toh, K., Srinivasan, D., Wong, K., Low, S.Q.: An empirical comparison of nine pattern classifiers. IEEE Transactions on Systems, Man, and Cybernetics, Part B: Cybernetics 35(5), 1079-1091 (2005)

15. Abdullah, M.R.B., Toh, K., Srinivasan, D.: A framework for empirical classifiers comparison. In: 1st IEEE Conference on Industrial Electronics and Applications, art. no. 4026002 (2006)

16. Asuncion, A., Newman, D.J.: UCI Machine Learning Repository. University of California, School of Information and Computer Science. Irvine, CA, U. S. A (2007), http://www.ics.uci.edu/ mlearn/MLRepository.html

17. Demsar, J.: Statistical comparisons of classifiers over multiple data sets. Journal of Machine Learning Research 7, 1-30 (2006)

18. Wolpert, D.H., Macready, W.G.: No free lunch theorems for optimization. IEEE Transactions on Evolutionary Computation 1(1), 67-82 (1997)

19. Wolpert, D.H.: The supervised learning no-free-lunch theorems. In: Proceedings of the 6th Online World Conference on Soft Computing in Industrial Applications (2001) 